\title{
Charge accumulation on a Luttinger liquid
}

\author{
Jason Alicea, ${ }^{*}$ Cristina Bena, ${ }^{\dagger}$ and Leon Balents ${ }^{\ddagger}$ \\ Physics Department, University of California, Santa Barbara, California 93106, USA
}

Matthew P. A. Fisher ${ }^{\S}$

Kavli Institute for Theoretical Physics, University of California, Santa Barbara, California 93106, USA

(Received 9 December 2003; published 28 April 2004)

\begin{abstract}
The average charge $Q$ on a quantum wire, modeled as a single-channel Luttinger liquid (LL), connected to metallic leads and coupled to a gate is studied theoretically. We find that the behavior of the charge as the gate voltage $\widetilde{V}_{G}$ varies depends strongly on experimentally adjustable parameters (length, contact transmission, temperature, ... ). When the intrinsic backscattering at the contacts is weak (i.e., the conductance is close to $2 e^{2} / h$ at high temperature), we predict that this behavior should be described by a universal function. For short such wires, the charge increases roughly linearly with $\widetilde{V}_{G}$, with small oscillations due to quantum interference between electrons scattered at the contacts. For longer wires at low temperature, Coulomb blockade behavior sets in, and the charge increases in steps. In both limits $\partial Q / \partial \widetilde{V}_{G}$, which should characterize the linear-response conductance, exhibits periodic peaks in $\widetilde{V}_{G}$. We show that due to Coulomb interactions the period in the former limit is twice that of the latter, and describe the evolution of the peaks through this crossover. The study can be generalized to multichannel LL's, and may explain qualitatively the recent observation by Liang et al. [Phys. Rev. Lett. 88, 126801 (2002)] of a four-electron periodicity for electron addition in single-walled carbon nanotubes.
\end{abstract}

DOI: 10.1103/PhysRevB.69.155332

PACS number(s): 73.23.Hk, 71.10.Pm

\section{INTRODUCTION}

The conductance of metallic single-walled carbon nanotubes has been shown to depend strongly on the nature of the contacts between the nanotube and the leads. In a typical experimental setup a bias voltage is applied across a nanotube connected to metallic leads, while a gate voltage applied to a third electrode acts as a chemical potential and modulates the charge on the nanotube. ${ }^{1-5}$ When the contacts between the nanotube and the leads are poor, Coulomb blockade behavior sets in, and the conductance exhibits a series of sharp peaks as the gate voltage increases. ${ }^{2,5,6}$ In contrast, the conductance of devices with near-perfect contacts is close to the theoretical maximum of $4 e^{2} / h$ for all gate voltages, with small quasiperiodic oscillations due to Fabry-Perot electron interference. $^{3}$

An interesting question that arises from these experiments is how the conductance of the system evolves in between these limits. Simple arguments indicate that the conductance undergoes a change in periodicity between the Coulomb blockade regime and the Fabry-Perot limit. Deep in the Fabry-Perot limit, based on a noninteracting picture in which each conducting channel is approximately independent, one expects one electron per channel (i.e., four for a nanotube) to be added to the wire per period of conductance oscillation. In the Coulomb blockade limit, peaks occur upon each electron addition process. This reasoning is in accord with recent experiments, ${ }^{1,3}$ in which transport measurements were performed on devices exhibiting a broad range of lowtemperature conductances. These experiments confirm the Fabry-Perot picture in the most conducting samples, regular Coulomb blockade behavior in the least conducting ones, and observed an interesting clustering of peaks into groups of four in an intermediate limit. ${ }^{1}$

Theoretically, there are two qualitative issues brought up by these experiments. First, why should the simple FabryPerot type structure obtained from a noninteracting quasiparticle scattering approach apply even in the highly conducting samples? This experimental result is somewhat surprising, since both theoretical expectation and numerous experiments indicate that Coulomb effects in nanotubes modify them from Fermi to Luttinger liquids, and in particular should not have electronlike quasiparticles! This question was addressed already in Ref. 7, where it was demonstrated that, for highly conducting samples, the behavior expected from Luttinger liquid (LL) theory (at least at low source-drain bias) is qualitatively indistinguishable from the naive quasiparticle scattering prediction. With this understood, the second issue raised is the nature of the crossover from this Fabry-Perot limit to the Coulomb blockade. Qualitatively, one would like to understand how the positions of the peaks evolve between the two regimes. This key qualitative issue of how the center of the peaks evolve is the focus of our work. Of course, the full universal crossover is interesting, but is more quantitative than qualitative.

A proper theoretical treatment of this evolution would require calculating the conductance of nanotube devices with arbitrary contact resistances. While this has been done perturbatively in the case of near-perfect contacts, ${ }^{7}$ nonperturbative techniques are required for intermediate contact resistances. Finding the conductance in the crossover regime is therefore of considerable difficulty. For low bias voltages, however, the qualitative features of the conductance should be manifested in the derivative of the average charge on the nanotube with respect to the gate voltage. This can be determined from an equilibrium calculation of the free energy and 


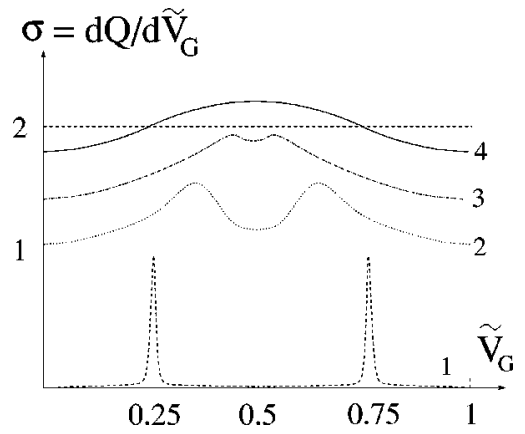

FIG. 1. The qualitative features of the crossover between good and poor contacts. The derivative of the charge with respect to the gate voltage (or the conductance) is depicted schematically for various values of the backscattering strength at the contacts. Curve 1 represents the Coulomb blockade situation, and curves 2 and 3 depict progressively smaller backscattering. Curve 4 illustrates the Fabry-Perot oscillations present in the low backscattering limit.

is consequently more tractable. Even with this simplification the problem is nontrivial, so we use Feynman's variational principle $^{8,9}$ to calculate the free energy and subsequently the charge. Since there is both experimental ${ }^{4}$ and theoretical ${ }^{10,11}$ evidence that carbon nanotubes behave as (LLs) (Ref. 12), we consider more generally the average charge on a singlechannel LL (with spin) with arbitrary electron-electron interaction strength. Our results can be generalized to multichannel LL's and therefore can be applied to nanotubes, which have two channels of conduction near the Fermi energy.

As expected, ${ }^{13}$ in the Coulomb blockade regime, when the contact resistance is large, we find that the charge increases discontinuously in steps whenever the gate voltage (in units in which two electrons are added to the wire whenever $\widetilde{V}_{G}$ is increased by one unit) $\widetilde{V}_{G}=(2 n+1) / 4$, where $n$ is an integer. The discontinuous character of these charge jumps is an artifact of our variational method and translates into $\delta$-function peaks in $\sigma=d Q / d \widetilde{V}_{G}$. The charge should be a continuous function of $\widetilde{V}_{G}$, and the height of the peaks in $\sigma$ should be finite. The variational method predicts accurately the locations of these peaks, but not their heights. If we correct for this artifact, $\sigma$ should behave qualitatively as depicted in curve 1 in Fig. 1. Despite the spin degeneracy, each peak corresponds to the addition of a single electron to the wire. Indeed, in this case single electrons can enter the LL only when they acquire the additional energy required to overcome the Coulomb repulsion energy and in this regime the periodicity of the peaks is $\delta \widetilde{V}_{G}=1 / 2$.

Although the method we use has the advantage of allowing us to consider arbitrary contact resistances, it yields unphysical results when the contact resistance is low. In this regime we resort instead to perturbation theory. In this limit we find that the effects of the electron-electron interactions are small. The spin-up and spin-down electrons propagate independently of each other so that two electrons are added to the LL per Fabry-Perot oscillation in $\sigma$. Consequently, $\sigma$ exhibits a broad sinusoidal oscillation ${ }^{14}$ with a period $\widetilde{V}_{G}$ $=1$, which is twice as large as the period in the Coulomb blockade regime. This behavior is represented by the curve 4 in Fig. 1.

The aim of this paper is to address how the crossover between the Coulomb blockade and the nearly perfect contacts regime takes place. One possibility is that as the contact resistance decreases, the Coulomb blockade peaks remain fixed in position but broaden asymmetrically so that they eventually combine to form the Fabry-Perot oscillations. A second possibility is that the Coulomb blockade peaks shift in position as they broaden and at some point collapse into each other to form the Fabry-Perot peaks. We find the latter possibility to be the case, and we illustrate it schematically in Fig. 1, which contains a sketch of $\sigma=d Q / d \widetilde{V}_{G}$ (or the conductance $G)$ as a function of $\widetilde{V}_{G}$ for different contact resistances. As the contact resistance decreases, we find that the peaks begin to shift toward the nearest half-integer value of $\widetilde{V}_{G}$ and the magnitude of the charge jump diminishes. Correcting for the discontinuity in the charge, $\sigma$ should evolve qualitatively as shown in Fig. 1 (curves 2 and 3) when the contact resistance decreases.

It should be stressed that in the limit of weak backscattering at the contacts, this crossover is universal. This follows from general renormalization group reasoning. It is well known that, for a spin-1/2 LL with full spin-rotational invariance (and this generalizes to the LL-Fermi liquid contacts considered here), there is a single relevant impurity backscattering operator. Hence, if the strength of the backscattering at each contact is weak and they are well separated from one another, all other effects of the imperfections of the contacts scale rapidly to zero on the scale of wire length. Hence, the behavior of such samples should be well described by a model containing only the ideal LL Hamiltonian and this leading backscattering operator at each contact. In particular, then, the charge $Q$ on the wire is a universal function of the interaction strength $g$, the renormalized backscattering strength $\sim u L^{1-g}$, where $L$ is the length of the wire, the gate voltage $\widetilde{V}_{G}$, the thermal length $k_{B} T L / \hbar v_{F}$, etc. This still contains many parameters that will affect the quantitative form of this crossover, but we expect our results for the evolution of the peak positions to be much more robust.

We establish the validity of our technique in Sec. II, where we apply Feynman's variational principle to calculate the free energy of an infinite, spinless LL with a single impurity. In Sec. III A, we discuss the variational solution for the charge on a spinless LL connected to semi-infinite leads. A similar analysis is performed in Sec. III B where we take into account spin. Finally, our results are summarized in Sec. IV.

\section{INFINITE LUTTINGER LIQUID WITH A SINGLE IMPURITY}

In this section we use Feynman's variational principle to calculate the free energy for an infinite, spinless Luttinger with an interaction parameter $g$ describing the strength of the interactions and a single impurity of scattering strength $u$. Although the free energy for this system can be computed exactly, ${ }^{15}$ applying the variational principle here allows us to 
establish the validity of this technique by comparing our results to the well-known renormalization group scaling results. ${ }^{16,17}$

We begin by writing an effective bosonized action ${ }^{16}$ for this system,

$$
S=\beta \sum_{\omega_{n}} \frac{\left|\omega_{n}\right|}{\pi g}\left|\theta_{0}\left(\omega_{n}\right)\right|^{2}-u \int_{0}^{\beta} d \tau \cos \left[2 \theta_{0}(\tau)\right],
$$

where $\theta_{0}$ represents $\theta(x)$ evaluated at the impurity site. The bosonic field $\theta$ and its dual $\phi$ are related to the right- and left-moving fermionic fields $\psi_{R / L}$ via the transformation $\psi_{R / L} \sim e^{i(\phi \pm \theta)}$. The Fourier-transform conventions we use throughout are

$$
\begin{gathered}
\theta\left(\omega_{n}\right)=\frac{1}{\beta} \int_{0}^{\beta} d \tau e^{i \omega_{n} \tau} \theta(\tau), \\
\theta(\tau)=\sum_{\omega_{n}} e^{-i \omega_{n} \tau} \theta\left(\omega_{n}\right) .
\end{gathered}
$$

We use nonstandard conventions for simplicity so that both $\theta(\omega)$ and $\theta(\tau)$ are dimensionless.

Determining the free energy for this system is nontrivial due to the presence of the cosine term in the effective action. We therefore approximate the free energy using Feynman's variational principle, ${ }^{8,9}$ which states that for some trial action $S^{\prime}$, the exact free energy obeys the following inequality:

$$
F<-\frac{1}{\beta} \ln Z^{\prime}+\frac{1}{\beta}\left\langle S-S^{\prime}\right\rangle_{S^{\prime}} \equiv F_{v},
$$

where $Z^{\prime}$ is the partition function corresponding to $S^{\prime}\left(Z^{\prime}\right.$ $=\int \mathcal{D} \theta e^{-S^{\prime}}$ ). The trial action $S^{\prime}$ is chosen to have a tractable form and to depend on variational parameters that are determined by minimizing the variational free energy $F_{v}$. The resulting variational free energy will be the best estimate for the exact free energy given the form of the trial action used.

In our case, the most general tractable trial action is quadratic in $\theta$, so we take our trial action to be

$$
S^{\prime}=\beta \sum_{\omega_{n}}\left(\lambda+\frac{\left|\omega_{n}\right|}{\pi g}\right)\left|\theta\left(\omega_{n}\right)\right|^{2},
$$

where $\lambda$ is a non-negative, frequency-independent variational parameter. This form can be obtained by expanding the cosine term in the effective action to second order in $\theta$ and replacing the scattering strength $u$ by an effective scattering strength $\lambda / 2$. This turns out to be the most general quadratic action that one needs to consider. Even if one takes into account an explicit frequency dependence for $\lambda$, the solution that minimizes the free energy is frequency independent.

Computing the variational free energy using Eq. (4) and setting $\partial F_{v} / \partial \lambda=0$, we find in the zero-temperature limit that the free energy is minimized when $\lambda$ satisfies the following equation:

$$
\lambda=2 u\left(\frac{\lambda}{\lambda+\epsilon_{0} /(\pi g)}\right)^{g} .
$$

where $\epsilon_{0}$ is a high-energy cutoff. For $g \geqslant 1$, Eq. (5) is satisfied only when $\lambda=0$. Thus the variational free energy is minimized by choosing a trial action which completely neglects the effects of scattering. For $g>1$ this result is consistent with renormalization group arguments, ${ }^{16}$ which state that $u$ is irrelevant for the case of attractive interactions and that the scattering strength is therefore renormalized to zero at energies much smaller than $\epsilon_{0}$. However, when $g=1$ the system reduces to a one-dimensional free fermion gas impinging on a barrier of height $u$. In this case the scattering strength is marginal, and the physics of the system (the value of the conductance, etc.) should depend on the scattering strength, which our variational technique fails to predict. For $g<1$, there exists a nontrivial solution to Eq. (5) given by

$$
\lambda \approx 2 u\left(\frac{2 \pi g u}{\epsilon_{0}}\right)^{g /(1-g)}
$$

that minimizes the free energy. It follows from Eq. (6) that $\lambda$ increases from 0 at $g=1$ to $2 u$ at $g=0$ so that the effective scattering strength increases with the strength of the interactions. This behavior is also consistent with renormalization group arguments since $u$ is relevant in this range of $g$. For this simple system, our variational technique therefore reproduces the well-known scaling results ${ }^{16}$ except in the case $g$ $=1$.

\section{FINITE-SIZE LUTTINGER LIQUID CONNECTED TO LEADS AND COUPLED TO A GATE}

\section{A. Spinless fermions}

In this section we calculate the charge on a spinless LL of length $2 L$ and interaction parameter $g$ connected to two semi-infinite leads characterized by an interaction parameter $g_{L}$. For simplicity, we assume that the Fermi velocity $v$ is uniform in the LL and the leads. The contacts with the leads at positions $x= \pm L$ are modeled as impurities of equal scattering strength $\tilde{u}$, measured in units of $\epsilon_{L}=v / 2 \pi L g^{2}$. A gate voltage $\widetilde{V}_{G}$ is applied to the LL, where $\widetilde{V}_{G}$ is expressed in units of $\pi^{2} \epsilon_{L}$ so that one electron is added to the LL when $\widetilde{V}_{G} \rightarrow \widetilde{V}_{G}+1$.

The effective action for this system is

$$
\begin{aligned}
\frac{S}{\epsilon_{L} \beta}= & \sum_{\omega_{n}} \sum_{a= \pm}\left|\theta_{a}\left(\omega_{n}\right)\right|^{2} \mathcal{K}_{a}\left(\omega_{n}\right)-\sqrt{2} \pi \widetilde{V}_{G} \theta_{-}(0) \\
& -\frac{\tilde{u}}{\beta} \int_{0}^{\beta} d \tau \sum_{\zeta= \pm 1} \cos \left[\sqrt{2} \theta_{+}(\tau)+\zeta \sqrt{2} \theta_{-}(\tau)\right],
\end{aligned}
$$

where $\theta_{ \pm}=[\theta(x=L) \pm \theta(x=-L)] / \sqrt{2}$ and $\theta_{-}(0)$ represents $\theta_{-}\left(\omega_{n}=0\right)$. The functions $\mathcal{K}_{ \pm}(\omega)$ appearing in Eq. (7) are given by

$$
\mathcal{K}_{ \pm}(\omega)=\frac{|\omega|}{2 \pi \epsilon_{L}}\left\{\frac{1}{g_{L}}+\frac{1}{g}\left[\tanh \left(\frac{|\omega|}{2 \pi \epsilon_{L} g}\right)\right]^{ \pm 1}\right\} .
$$

To calculate the variational free energy, we take a trial action of the form 


$$
\begin{aligned}
\frac{S^{\prime}}{\epsilon_{L} \beta}= & \sum_{\omega_{n}} \sum_{a= \pm}\left|\theta_{a}\left(\omega_{n}\right)\right|^{2}\left[\mathcal{K}_{a}\left(\omega_{n}\right)+\lambda_{a}\right] \\
& -\sqrt{2} \pi\left[\left(\tilde{V}_{G}+\lambda_{G}\right) \theta_{-}(0)+\mu_{+} \theta_{+}(0)\right],
\end{aligned}
$$

and assume that the variational parameters $\lambda_{ \pm}$are frequency independent and non-negative. If one allows $\lambda_{ \pm}$to depend on frequency, one finds as in the single-impurity case that the free energy is minimized when $\lambda_{ \pm}$are frequency independent. The quadratic terms in Eq. (9) can be obtained by expanding the cosine term in the effective action to second order and replacing $\tilde{u}$ by $\lambda_{ \pm} / 2$, so we can once more interpret $\lambda_{ \pm}$as effective scattering strengths. To obtain the linear terms in Eq. (9), we shift $\widetilde{V}_{G}$ by a variational parameter $\lambda_{G}$ and introduce another variational parameter $\mu_{+}$that multiplies $\theta_{+}(0)$. The inclusion of the latter term, which does not appear in the effective action in Eq. (7), is necessary in order to preserve the invariance of the original Hamiltonian under the addition of an extra unit of charge.

The variational free energy computed from the trial action in Eq. (9) is given in Eq. (A1) in Appendix A. Setting the derivatives of the free energy with respect to the variational parameters to zero, we find that the free energy will be minimized when $\lambda_{ \pm}$satisfy the following equation:

$$
\begin{aligned}
\lambda_{+}= & \lambda_{-} \equiv \lambda=\zeta \gamma \lambda^{\Delta} e^{-I(\lambda)} \cos \left[\pi \widetilde{V}_{G}\right. \\
& \left.+\eta \sqrt{\gamma^{2} \lambda^{2 \Delta} e^{-2 I(\lambda)}-\lambda^{2}}\right]
\end{aligned}
$$

where $\cos \left(\pi \mu_{+} / \lambda\right) \equiv \zeta= \pm 1, \quad \eta= \pm 1, \quad$ and $\Delta=2 g_{L} g /\left(g_{L}\right.$ $+g)$. The dimensionless parameter that determines the behavior of the system is $\gamma=2 \tilde{u}\left(\pi \Delta \epsilon_{L} / \epsilon_{0}\right)^{\Delta}$, and in the zerotemperature limit $I(\lambda)$ is given by

$$
I(\lambda)=\int_{0}^{\infty} d x\left[\sum_{a= \pm} \frac{1}{\mathcal{K}_{a}\left(2 \pi \epsilon_{L} x\right)+\lambda}-\frac{1}{x / \Delta+\lambda / 2}\right] .
$$

The signs of $\zeta$ and $\eta$ depend on the gate voltage and should be chosen such that the free energy is minimized and $\lambda$ is non-negative. To satisfy these conditions, we choose $\zeta=$ +1 if $-1 / 2<\widetilde{V}_{G}<1 / 2$ and change the sign of $\zeta$ whenever $\widetilde{V}_{G} \rightarrow \widetilde{V}_{G}+1$. Also, we take $\eta=-1$ if $0<\widetilde{V}_{G}<1 / 2$ and change the sign of $\eta$ whenever $\widetilde{V}_{G} \rightarrow \widetilde{V}_{G}+1 / 2$.

Since the gate voltage acts as a chemical potential, the average charge on the LL (in units of the electron charge) is $Q=-\left(\partial F_{v} / \partial \widetilde{V}_{G}\right) /\left(\pi^{2} \epsilon_{L}\right)$. When the free energy is minimized, the charge can be written in terms of $\lambda$ as

$$
Q(\lambda)=\frac{\widetilde{V}_{G}+\lambda_{G}}{1+\lambda}=\widetilde{V}_{G}+\frac{\eta}{\pi} \sqrt{\gamma^{2} \lambda^{2 \Delta} e^{-2 I(\lambda)}-\lambda^{2}} .
$$

To determine the charge, one must therefore find the solution to Eq. (10) that minimizes the free energy.

In the limit $\gamma \gg 1$ we can solve Eq. (10) analytically by assuming that $\lambda$ is large and roughly independent of $\widetilde{V}_{G}$. The dominant contribution to the integral in $I(\lambda)$ then comes from the region where $x \gg 1$. We therefore approximate $\mathcal{K}_{ \pm}\left(2 \pi \epsilon_{L} x\right) \approx 2 x / \Delta$ and take $I(\lambda) \approx 0$. With these assump- tions we find that $\lambda=0$ is the only solution when $\Delta \geqslant 1$. For $\Delta<1$, which is the relevant physical situation since we are interested in repulsive interactions, the free energy is minimized by a nontrivial value of $\lambda$. The charge is then

$$
Q \approx n+\eta\left[\frac{n-\widetilde{V}_{G}}{\gamma^{1 /(1-\Delta)}}\right]
$$

where $n$ is the closest integer to $\widetilde{V}_{G}$. This solution corresponds to the Coulomb blockade limit since the charge increases in steps whenever $\widetilde{V}_{G}$ equals a half integer. We note that the charge jumps discontinuously at these values of $\widetilde{V}_{G}$, resulting in the emergence of $\delta$-function peaks in $\sigma$. This discontinuity is an artifact of the variational method and can be traced back to the replacement of the cosine term in the effective action by quadratic terms in our trial action. The effect of this approximation is to suppress tunneling events between different minima in the cosine potential, which correspond to quantum-mechanical mixing of the two nearly degenerate charge states of the LL. These instantons become important when the gate voltage is near a half integer and would cause the charge to increase smoothly in this region. Since we ignore such events in our calculations, the charge jumps discontinuously. Intuitively, the charge should be a continuous function of $\widetilde{V}_{G}$, and the peaks in $\sigma$ should be rounded and have finite height.

For smaller values of $\gamma$, we obtain the charge by numerically solving Eq. (10) for the value of $\lambda$ that minimizes the free energy. To illustrate the evolution of the charge, we plot $Q$ and its derivative $\sigma=\partial Q / \partial \widetilde{V}_{G}$ as functions of $\widetilde{V}_{G}$ for different values of $\gamma$, focusing on a system at zero temperature with Fermi-liquid leads $\left(g_{L}=1\right), g=0.25$, and $\epsilon_{L} / \epsilon_{0}$ $=2.55 \times 10^{-3}$. The solid lines in Figs. 2 and 3 represent $Q$ and $\sigma$ evaluated at $\gamma=2.37$ and 0.8 , respectively. For comparison, these quantities evaluated at $\gamma=0$ are shown as the dashed lines. In Figs. 2 and 3(b), we do not show the full peaks in $\sigma$ at $\widetilde{V}_{G}=1 / 2$ to emphasize the structure away from $\widetilde{V}_{G}=1 / 2$. These figures illustrate that the magnitude of the discontinuous jump in the charge $\delta Q$ diminishes as $\gamma$ decreases. Additionally, the slope of the charge away from halfinteger values of $\widetilde{V}_{G}$ increases from 0 toward 1 .

As $\gamma$ decreases further, the behavior of the variational solution for the charge depends on whether $g_{L}$ is greater than or less than $1 / 2$. For $g_{L}<1 / 2, \delta Q$ decreases smoothly to zero as $\gamma \rightarrow 0$. This is illustrated in Fig. 4(b), which contains $\delta Q$ versus $\gamma$ when $g_{L}=1 / 4$. Apart from the discontinuity in the charge at half-integer values of $\widetilde{V}_{G}$, in the low backscattering limit the variational method produces the expected Fabry-Perot oscillations.

With $g_{L} \geqslant 1 / 2, \delta Q$ drops abruptly to zero when $\gamma$ equals a critical value denoted by $\gamma_{c}$. This feature is illustrated in Fig. 4(a), where $\gamma_{c} \approx 0.78$. Below $\gamma_{c}$, the variational method predicts unphysical behavior in the charge that, in particular, is inconsistent with a perturbative calculation of the charge in the limit $\gamma \ll 1$. Details of the variational solution in this regime will therefore be deferred to Appendix B. The variational method predicts the presence of two sharp peaks in $\sigma$ 


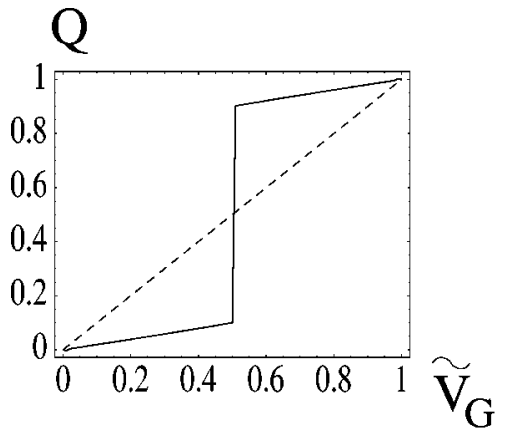

a)

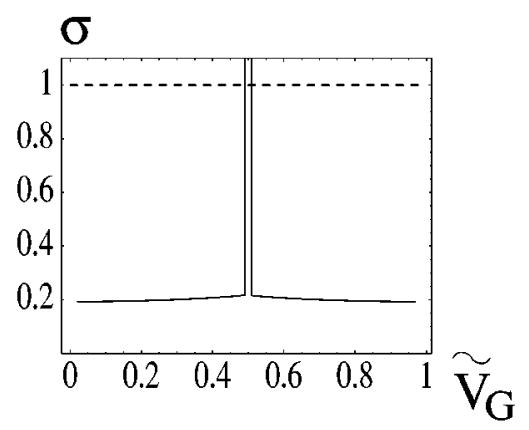

b)
FIG. 2. The charge $\mathrm{Q}$ and $\sigma=\partial Q / \partial \widetilde{V}_{G}$ as functions of the gate voltage for a system with $g_{L}=1, \quad g=0.25, \quad \epsilon_{L} / \epsilon_{0}=2.55 \times 10^{-3}, \quad$ and $\gamma$ $=2.37$. The discontinuity in the charge at $\widetilde{V}_{G}$ $=1 / 2$ is an artifact of the variational technique that arises from our replacing the cosine term in the effective action by quadratic terms in our trial action. This results in $\delta$-function peaks in $\sigma$ which should instead be rounded and have finite height. For clarity, we do not show the full peak in $\sigma$ at $\widetilde{V}_{G}=1 / 2$ in (b). per period rather than the single broad peak expected from the Fabry-Perot oscillations. Figure 5 contains $Q$ and $\sigma$ for a system with $g_{L}=1$ and $\gamma<\gamma_{c}$. The double-peak structure that emerges is a consequence of the free energy being minimized when $\lambda=0$ in the region between these peaks. We attribute this shortcoming of the variational technique to the method failing to capture the analytic terms in the free energy, which presumably dominate in this regime. In contrast, when $g_{L}<1 / 2$ even the lowest-order term in perturbation theory diverges, so there are no analytic terms in the free energy. This explains why the variational technique yields reasonable results when $g_{L}<1 / 2$ for arbitrary $\gamma$ but fails in the low backscattering limit when $g_{L} \geqslant 1 / 2$.

In the limit $\gamma \ll 1$, the charge can instead be calculated perturbatively in $\tilde{u}$ when $g_{L}>1 / 2$. To second order in $\tilde{u}$, we find

$$
Q=\widetilde{V}_{G}-A \gamma^{2} \sin \left(2 \pi \widetilde{V}_{G}\right),
$$

where $A$ is a positive constant. It follows that $\sigma$ is roughly constant, exhibiting small Fabry-Perot oscillations with periodicity $\delta \widetilde{V}_{G}=1$. Thus, although the variational method fails in the low backscattering limit, the expected behavior is captured perturbatively.

In summary, we have shown that for a spinless, singlechannel LL the periodicity of $\sigma$ is the same in the low backscattering and Coulomb blockade limits. After correcting for the artifacts of the variational technique, we find that the Coulomb blockade peaks in $\sigma$ simply broaden symmetrically as the backscattering strength decreases, eventually evolving into the broad Fabry-Perot peaks in the low backscattering limit.

\section{B. Spinful fermions}

We now repeat the calculations of Sec. III A taking into account spin. It is convenient to decompose the system into charge and spin sectors via a change of basis to fields $\theta_{\rho / \sigma}$ $=\left(\theta_{\uparrow} \pm \theta_{\downarrow}\right) / \sqrt{2}$. Since the interactions only affect the charge sector, the spin sector in both the leads and in the LL is characterized by an interaction parameter of $g^{\sigma}=1$. The charge sector is characterized by an interaction parameter $g_{L}$ in the leads and $g$ in the LL.

When spin is taken into account, the effective action in Eq. (7) is generalized to

$$
\begin{aligned}
\frac{S}{\epsilon_{L} \beta}= & \sum_{\omega_{n}, a}\left|\theta_{a}\left(\omega_{n}\right)\right|^{2} \mathcal{K}_{a}\left(\omega_{n}\right)-2 \pi \widetilde{V}_{G} \theta_{\rho-}(0) \\
& -\frac{\tilde{u}}{\beta} \int_{0}^{\beta} d \tau \sum_{\zeta_{1}, \zeta_{2}} \cos \left[\theta_{\rho+}(\tau)+\zeta_{1} \theta_{\rho-}(\tau)+\zeta_{2} \theta_{\sigma+}(\tau)\right. \\
& \left.+\zeta_{1} \zeta_{2} \theta_{\sigma-}(\tau)\right]
\end{aligned}
$$

where $a=\rho \pm, \sigma \pm$ are charge and spin indices, respectively, and $\zeta_{1,2}= \pm 1$. The functions $\mathcal{K}_{a}\left(\omega_{n}\right)$ are defined by

$$
\mathcal{K}_{a}(\omega)=\frac{|\omega|}{2 \pi \epsilon_{L}}\left\{\frac{1}{g_{L}^{a}}+\frac{1}{g^{a}}\left[\tanh \left(\frac{|\omega|}{2 \pi \epsilon_{L} g^{a}}\right)\right]^{\zeta_{a}}\right\},
$$

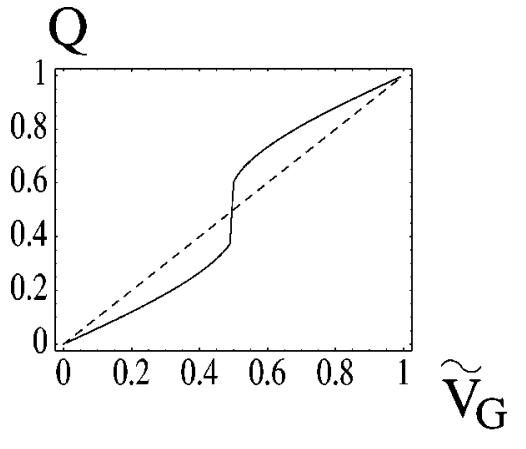

a)

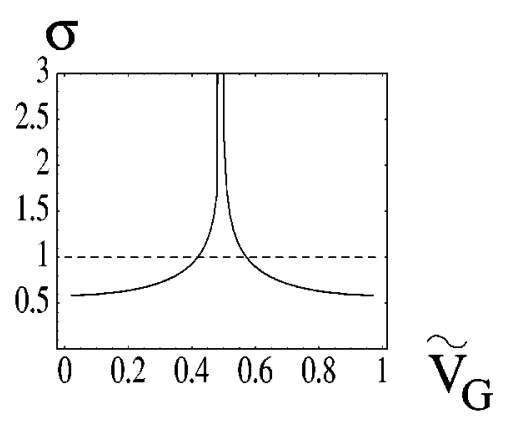

b)
FIG. 3. The charge $\mathrm{Q}$ and $\sigma=\partial Q / \partial \widetilde{V}_{G}$ as functions of the gate voltage for a system with $g_{L}=1, \quad g=0.25, \quad \epsilon_{L} / \epsilon_{0}=2.55 \times 10^{-3}, \quad$ and $\gamma$ $=0.8$. As $\gamma$ decreases the discontinuous jump in the charge at $\widetilde{V}_{G}=1 / 2$ diminishes, and the slope of the charge away from $\widetilde{V}_{G}=1 / 2$ increases. The sharp peak in $\sigma$ at $\widetilde{V}_{G}=1 / 2$ is not shown in (b) for clarity. 


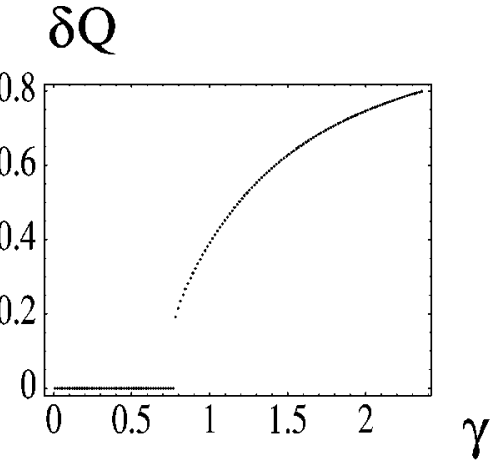

a)

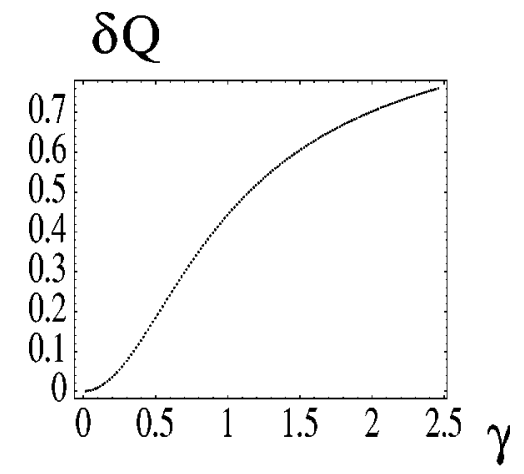

b) where $g_{L}^{\rho^{ \pm}}=g_{L}, \quad g^{\rho^{ \pm}}=g, \quad g_{L}^{\sigma^{ \pm}}=g^{\sigma \pm}=1, \quad \zeta_{\rho / \sigma+}=1$, and $\zeta_{\rho / \sigma^{-}}=-1$. We assume a trial action of the form

$$
\begin{aligned}
\frac{S^{\prime}}{\epsilon_{L} \beta}= & \sum_{\omega_{n}, a}\left|\theta_{a}\left(\omega_{n}\right)\right|^{2}\left[\mathcal{K}_{a}\left(\omega_{n}\right)+\lambda_{a}\right]-2 \pi\left[\mu_{\rho+} \theta_{\rho+}(0)\right. \\
& \left.+\left(\widetilde{V}_{G}+\lambda_{G}\right) \theta_{\rho-}(0)+\mu_{\sigma+} \theta_{\sigma+}(0)+\mu_{\sigma-} \theta_{\sigma-}(0)\right] .
\end{aligned}
$$

Here, $\lambda_{\rho / \sigma \pm}$ represent effective scattering strengths and $\lambda_{G}$, $\mu_{\rho+}$, and $\mu_{\sigma \pm}$ are additional variational parameters. The variational free energy computed from this trial action is given in Eq. (A2) in Appendix A. As in the spinless case, the free energy is minimized when the effective scattering strengths are equal, so we define $\lambda_{\rho / \sigma_{ \pm}} \equiv \lambda$.

As outlined in Appendix A, setting the derivatives of the free energy with respect to the variational parameters to zero leads to two sets of equations for the variational parameters that result in physical solutions for the charge. In the first set, the charge is given in terms of $\lambda$ by

$$
Q(\lambda)=2 \widetilde{V}_{G}+\frac{2 \eta}{\pi} \sqrt{\gamma^{\prime 2} \lambda^{\Delta+1} e^{-2 I^{\prime}(\lambda)}-\lambda^{2}}
$$

where $\lambda$ is a solution to

$$
\lambda=\zeta \gamma^{\prime} \lambda^{(\Delta+1) / 2} e^{-I^{\prime}(\lambda)} \cos \left[\frac{\pi Q(\lambda)}{2}\right]
$$

Here, $\gamma^{\prime}=2 \tilde{u} \Delta^{-1 / 2}\left(\pi \Delta \epsilon_{L} / \epsilon_{0}\right)^{(\Delta+1) / 2}$ is the dimensionless parameter that determines the behavior of the system, and

$$
I^{\prime}(\lambda)=\int_{0}^{\infty} \frac{d u}{2}\left[\sum_{a} \frac{1}{\mathcal{K}_{a}\left(2 \pi \epsilon_{L} u\right)+\lambda}-\frac{1}{u / \Delta+\lambda / 2}-\frac{1}{u+\lambda / 2}\right]
$$

in the zero temperature limit. The values of $\eta$ and $\zeta$ are the same as in the spinless case. In the second set of equations, the charge is determined from the following equation:

$$
\begin{aligned}
\pi \mid Q / 2 & -\widetilde{V}_{G} \mid \\
= & \gamma^{\prime} \lambda^{(\Delta+1) / 2} e^{-I^{\prime}(\lambda)\left|\cos \left(\frac{\pi Q}{2}\right)\right|} \\
& \times \mid \sin \left[\frac{1}{g^{2}} \sqrt{\gamma^{\prime 2} \lambda^{\Delta+1} e^{-2 I^{\prime}(\lambda)} \sin ^{2}\left(\frac{\pi Q}{2}\right)-\lambda^{2}}\right],
\end{aligned}
$$

where $\lambda$ is a function of the charge via

$$
\lambda(Q)=\left|\pi\left(Q / 2-\widetilde{V}_{G}\right) \tan (\pi Q / 2)\right| .
$$

To determine which set of equations leads to an absolute minimum of the free energy, we find numerically the minimum free energy solution to both sets and retain the one with the lower free energy.

In the Coulomb blockade regime where $\gamma^{\prime} \gg 1$, the charge is given by Eq. (18) when $n-1 / 4<\widetilde{V}_{G}<n+1 / 4$ and by Eq. (21) when $n+1 / 4<\widetilde{V}_{G}<n+3 / 4$, where $n$ is an integer. In

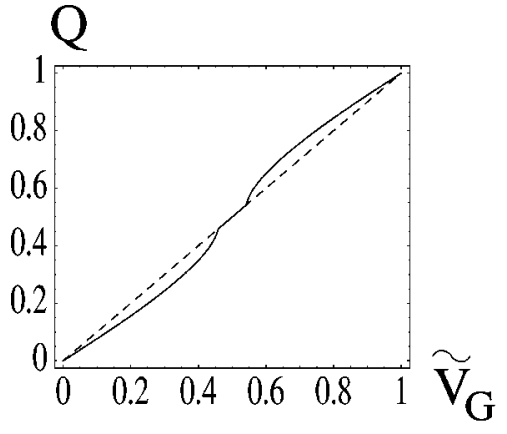

a)

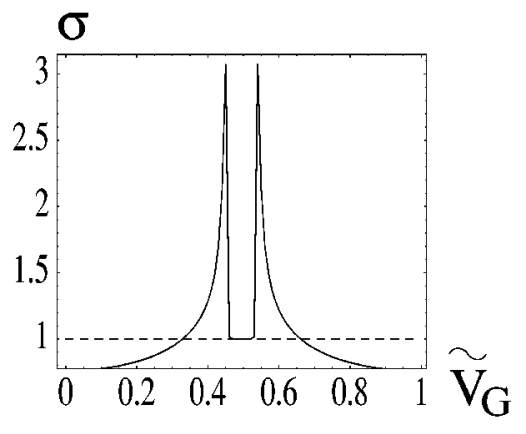

b)
FIG. 5. The charge $\mathrm{Q}$ and $\sigma=\partial Q / \partial \widetilde{V}_{G}$ as functions of the gate voltage for a system with Fermi-liquid leads $\left(g_{L}=1\right), \quad g=0.25, \epsilon_{L} / \epsilon_{0}$ $=2.55 \times 10^{-3}$, and $\gamma=0.47<\gamma_{c}$. The two peaks present in $\sigma$ arise from the free energy being minimized when $\lambda=0$ in the region between the peaks. This double-peak structure is an artifact due to the variational method failing to capture the analytic terms in the free energy. 


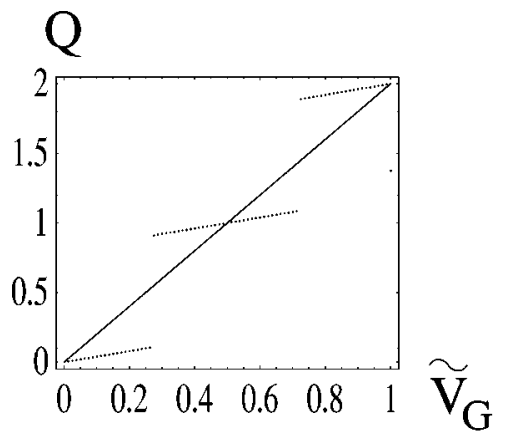

a)

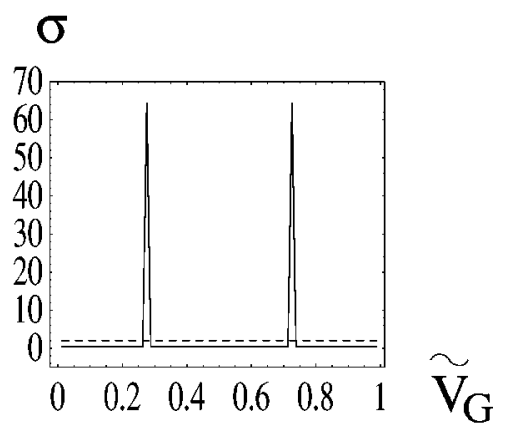

b) this limit the charge can be retrieved analytically by assuming $\lambda \gg 1$ and taking $I^{\prime}(\lambda) \approx 0$. As in the spinless case, we find nontrivial solutions for $\lambda$ only when $\Delta<1$. The charge in this range of $\Delta$ is given by

$$
Q \approx n+2 \eta^{\prime}\left[\frac{n / 2-\widetilde{V}_{G}}{\gamma^{\prime 2 /(1-\Delta)}}\right],
$$

where $n$ is the closest integer to $2 \widetilde{V}_{G}$ and $\eta^{\prime}=-1$ if 0 $<\widetilde{V}_{G}<1 / 4$ and changes sign whenever $\widetilde{V}_{G} \rightarrow \widetilde{V}_{G}+1 / 4$. Thus the charge increases in steps, jumping discontinuously by an amount $\delta Q \approx 1$ at half-integer values of $2 \widetilde{V}_{G}$. This discontinuity is again an artifact of our technique and results in $\delta$-function peaks in $\sigma$ rather than rounded peaks of finite height. These $\delta$-function peaks occur at half-integer values of $2 \widetilde{V}_{G}$, so the period of $\sigma$ in the Coulomb blockade regime is $\delta \widetilde{V}_{G}=1 / 2$.

As $\gamma^{\prime}$ decreases and the system moves away from the Coulomb blockade limit, the window of $\widetilde{V}_{G}$ inside of which the second set of equations yields the lowest free energy begins to shrink. The charge remains discontinuous at the end points of the window, causing the peaks in $\sigma$ to shift. Figures 6, 7, and 8 represent $\gamma^{\prime}=1.56,0.67$, and 0.58 , respectively, and illustrate how $Q$ and $\sigma$ evolve as $\gamma^{\prime}$ decreases. For our numerical data, we again focus on a system at zero temperature with Fermi-liquid leads, $g=0.25$, and $\epsilon_{L} / \epsilon_{0}=2.55 \times 10^{-3}$. To illustrate the nonlinear behavior in the charge more clearly, $Q-2 \widetilde{V}_{G}$ has been scaled by a factor of 3 in Fig. 7(a) and a factor of 4 in Fig. 8(a). In Fig. 8, the peaks in $\sigma$ have shifted significantly and appear at roughly $\widetilde{V}_{G}=0.4$ and 0.6 .

When $\gamma^{\prime}$ reaches a critical value $\gamma_{c}^{\prime}$, the first set of equations yields the lowest free energy for all $\widetilde{V}_{G}$, and the charge is given everywhere by Eq. (18). The discontinuous jump in the charge drops smoothly to zero at $\gamma_{c}^{\prime}$, and the charge remains continuous at smaller values of $\gamma^{\prime}$. For $\gamma^{\prime} \lesssim \gamma_{c}^{\prime}$, the variational method predicts unphysical behavior in the charge that is inconsistent with perturbation theory when $\gamma^{\prime} \ll 1$, so we defer details of the solution to Appendix B. In contrast to the spinless case, where the method was reliable for arbitrary $\gamma^{\prime}$ when $g_{L}<1 / 2$, the variational technique fails in this range of $\gamma^{\prime}$ for all $g_{L}$. This is consistent with the fact that in the spinful case the effective interaction parameter for the leads is $\left(g_{L}+1\right) / 2$, which is always greater than $1 / 2$. Figure 9 illustrates the unphysical behavior in the charge predicted by the variational method when $\gamma^{\prime}=0.33<\gamma_{c}^{\prime}$. For clarity, $Q-2 \widetilde{V}_{G}$ has been scaled by a factor of 30 in the charge. Nonlinear corrections to the charge are therefore quite small at this value of $\gamma^{\prime}$, and $\sigma$ is nearly constant. The variational method predicts two peaks in $\sigma$ per period rather than the single broad Fabry-Perot peak expected in this regime. This double-peak structure persists as $\gamma^{\prime}$ decreases further until $Q=2 \widetilde{V}_{G}$ and the peaks disappear altogether. As in the spinless case, we attribute this failure of the variational technique to the method neglecting analytic terms in the free energy. We therefore emphasize instead a perturbative calcu-

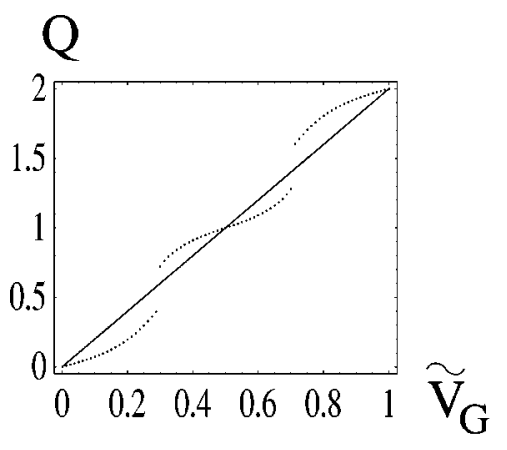

a)

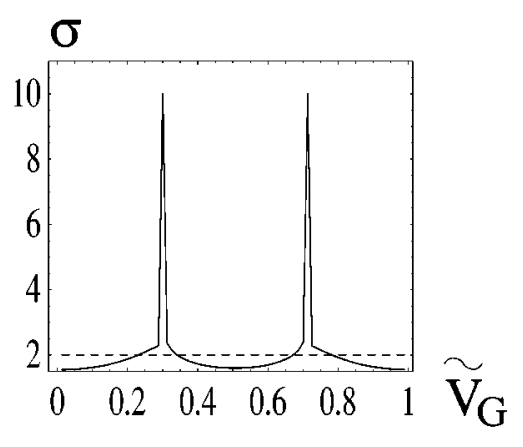

b)
FIG. 7. The charge $\mathrm{Q}$ and $\sigma=\partial Q / \partial \widetilde{V}_{G}$ as functions of the gate voltage for a system with $g_{L}=1, \quad g=0.25, \quad \epsilon_{L} / \epsilon_{0}=2.55 \times 10^{-3}$, and $\gamma^{\prime}$ $=0.67$. For clarity, we have scaled $Q-2 \widetilde{V}_{G}$ by a factor of 3 in the plot of the charge. The peaks in $\sigma$ are now located near $\widetilde{V}_{G}=0.3$ and 0.7 . 


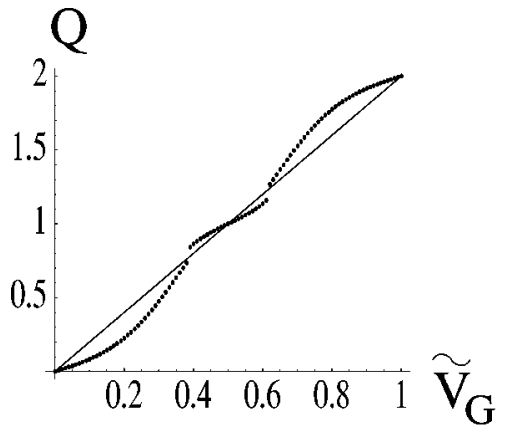

a)

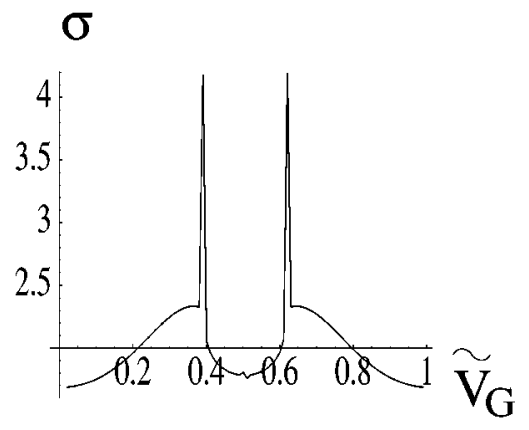

b) lation of the charge in the low backscattering limit. To second order in $\tilde{u}$, we find perturbatively that for any $g_{L}>0$,

$$
Q=2 \widetilde{V}_{G}-A^{\prime} \gamma^{\prime 2} \sin \left(2 \pi \widetilde{V}_{G}\right),
$$

where $A^{\prime}$ is a positive constant. The period of the FabryPerot oscillations is therefore $\delta \widetilde{V}_{G}=1$, which is the same as in the spinless case [see Eq. (14)].

In summary, we have shown that the period of $\sigma$ for a spinful LL increases by a factor of 2 as the system goes from the Coulomb blockade regime to the low backscattering limit. The numerical data in Figs. 6-8 along with the perturbative calculation of the charge in Eq. (24) demonstrate that once we correct for variational artifacts, this crossover in periodicity takes place as sketched schematically in Fig. 1. As the backscattering strength at the contacts decreases, the Coulomb blockade peaks shift in position as they broaden, and eventually combine to form the broad Fabry-Perot oscillations. As mentioned before, the physics behind this crossover in periodicity is that in the Coulomb blockade regime electrons are added one-by-one due to electron-electron interactions. In the low backscattering limit, however, the spin-up and spin-down electrons essentially propagate independently of one another, so that two electrons are added to the LL per period of the Fabry-Perot oscillations in $\sigma$.

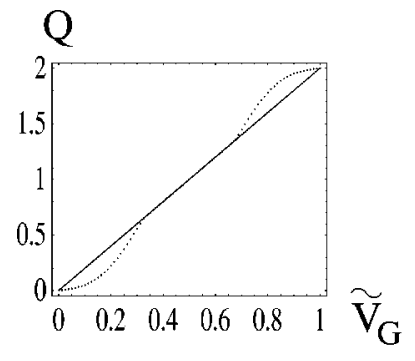

a)

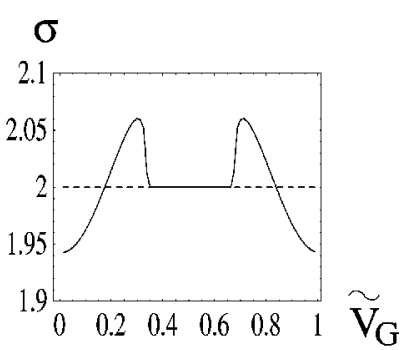

b)
FIG. 9. The charge $\mathrm{Q}$ and $\sigma=\partial Q / \partial \widetilde{V}_{G}$ as functions of the gate voltage for a spinful system with $g_{L}=1, g=0.25, \epsilon_{L} / \epsilon_{0}=2.55$ $\times 10^{-3}$, and $\gamma^{\prime}=0.33$, which is slightly below $\gamma_{c}^{\prime}$. To emphasize the nonlinear behavior in the charge, we have scaled $Q-2 \widetilde{V}_{G}$ by a factor of 30 in the plot of the charge. The double-peak structure in $\sigma$ is an unphysical artifact of the variational method that results from the technique neglecting analytic terms in the free energy.
FIG. 8. The charge $\mathrm{Q}$ and $\sigma=\partial Q / \partial \widetilde{V}_{G}$ as functions of the gate voltage for a system with $g_{L}=1, \quad g=0.25, \quad \epsilon_{L} / \epsilon_{0}=2.55 \times 10^{-3}$, and $\gamma^{\prime}$ $=0.58$, which is close to $\gamma_{c}^{\prime}$. For clarity, $Q$ $-2 \widetilde{V}_{G}$ has been scaled by a factor of 4 in the plot of the charge. The peaks in $\sigma$ continue to move toward $\widetilde{V}_{G}=1 / 2$ and now appear at roughly $\widetilde{V}_{G}$ $=0.4$ and 0.6 .

\section{DISCUSSION}

We discussed the average charge on finite-size spinless and spinful Luttinger liquids as a function of an applied gate voltage. As expected, in both cases the charge increases almost linearly with gate voltage when the contacts are good, while the charge increases in steps in the Coulomb blockade regime. In the spinless case, the derivative of the charge with respect to the gate voltage $\sigma$ has the same periodicity in both limits. When spin is taken into account, however, we showed that each Fabry-Perot peak begins to separate into two peaks as the contact resistance increases. As illustrated schematically in Fig. 1, the spacing between these peaks increases with the contact resistance until the Coulomb blockade limit is reached and the period of $\sigma$ is reduced by a factor of 2 . The physics behind this crossover is that when the contacts are good, electrons from the two spin channels essentially propagate independently of one another so that two electrons are added to the LL per period of the Fabry-Perot oscillations. In the Coulomb blockade regime, however, despite the spin degeneracy electrons are added to the LL one at a time due to electron-electron interactions, resulting in a reduction in periodicity.

The shifting of the peaks between these limits can be understood heuristically from a simple "Hartree" picture of free electron levels and a global charging energy (though it should be kept in mind that such a picture misses many details of the LL physics). In this framework, there is a difference in energy required to add an electron to a LL with odd versus even charge. If the number of electrons on the LL is odd, then in the ground state there will be an energy level occupied by a single spin. This energy level can then accommodate an additional electron with the opposite spin. In the ground state of a LL with an even number of electrons, however, each energy level is doubly occupied, so adding an electron requires additional kinetic energy relative to the LL with odd charge. Deep in the Coulomb blockade regime, this energy difference will be much less than the Coulomb energy required to add an electron. As the contact resistance decreases and the Coulomb interactions become less important, this energy difference can become appreciable. Since the gate voltage supplies the energy for electrons to tunnel onto the LL, these arguments imply that as the contact resistance increases the peaks in $\sigma$ should shift towards half-integer 
gate voltages where the charge is odd, which is consistent with what we find.

In an obvious generalization, we expect that if there are $n$ conduction channels, then the period should change by a factor of $2 n$, with the factor of 2 arising from spin. This crossover in periodicity should also appear in the behavior of the conductance. Specifically, rather than pairs of Coulomb blockade peaks combining to form the Fabry-Perot oscillations, we expect $2 n$ Coulomb blockade peaks to collapse onto each other in the low backscattering limit. Addressing how these peaks collapse, however, requires further investigation.

This crossover in periodicity can be used to interpret recent experimental data on carbon nanotubes, which have been shown to behave as two-channel LL's. ${ }^{4,10,11}$ As described in the Introduction, the conductance of nanotubes has been measured in devices with near-perfect contacts, ${ }^{3}$ in the Coulomb blockade limit, ${ }^{2,5}$ and more recently in an intermediate regime. ${ }^{1}$ When the contacts are good, we have seen that four electrons should be added to the nanotube per period of the Fabry-Perot oscillations in the conductance. As the contact resistance increases, corresponding to an increase in $\gamma^{\prime}$, each of these oscillations should begin to separate into four distinct peaks as the crossover begins to take place. The manner in which this takes place cannot be implied from our single-channel calculation, though a few possibilities can occur: all four peaks will appear simultaneously and then evolve and separate symmetrically; two peaks will arise, evolve and separate symmetrically, then each of them will split into two peaks; two peaks will arise and separate first, then when they reached a certain distance two more peaks will appear. The recent observation of a four-electron periodicity for electron addition by Liang et al. ${ }^{1}$ may be a manifestation of this crossover (see Fig. 1 in that reference). The conductance of their devices exhibited peaks grouped into clusters of four as the gate voltage increased. We note that their measurements are consistent with our prediction that the peaks shift in position as the contact resistance changes since adjacent conductance peaks within a given cluster are closer together than adjacent peaks in neighboring clusters. As the contact resistance increases further, the four peaks within each cluster should continue to separate and become sharper until the Coulomb blockade limit is reached and the period of the conductance is reduced by a factor of 4 .

\section{ACKNOWLEDGMENTS}

We thank Smitha Vishveshwara for many illuminating discussions and for contributions to the initial stages of this work. J.A. gratefully acknowledges support from an NSF Graduate Research Fellowship. L.B. was supported by the NSF through Grant No. DMR-9985255, and by the Sloan and Packard foundations. M.P.A.F. was supported by the NSF under grants No. DMR-0210790 and PHY-9907949.

\section{APPENDIX A: VARIATIONAL FREE ENERGY}

The variational free energy for the spinless LL computed using the trial action in Eq. (9) is

$$
\begin{aligned}
\frac{F_{v}}{\epsilon_{L}}= & \frac{\pi^{2}}{2}\left[\frac{\left(\widetilde{V}_{G}+\lambda_{G}\right)^{2}}{\left(1+\lambda_{-}\right)^{2}}-\frac{2 \widetilde{V}_{G}\left(\widetilde{V}_{G}+\lambda_{G}\right)}{1+\lambda_{-}}\right] \\
& +\frac{1}{2 \epsilon_{L} \beta} \sum_{\omega_{n}} \sum_{a= \pm} \ln \left[\frac{\epsilon_{L} \beta}{\pi}\left[\mathcal{K}_{a}\left(\omega_{n}\right)+\lambda_{a}\right]\right] \\
& -\frac{1}{2 \epsilon_{L} \beta} \sum_{\omega_{n}} \sum_{a= \pm} \frac{\lambda_{a}}{\lambda_{a}+\mathcal{K}_{a}\left(\omega_{n}\right)} \\
& -2 \tilde{u} \cos \left[\frac{\pi\left(\widetilde{V}_{G}+\lambda_{G}\right)}{1+\lambda_{-}}\right] \cos \left(\frac{\pi \mu_{+}}{\lambda_{+}}\right) \\
& \times \exp \left[-\frac{1}{2 \epsilon_{L} \beta} \sum_{\omega_{n}} \sum_{a= \pm} \frac{1}{\mathcal{K}_{a}\left(\omega_{n}\right)+\lambda_{a}}\right] .
\end{aligned}
$$

The variational free energy for the spinful LL corresponding to the trial action in Eq. (17) is

$$
\begin{aligned}
\frac{F_{v}}{\epsilon_{L}}= & \pi^{2}\left[\frac{\left(\widetilde{V}_{G}+\lambda_{G}\right)^{2}}{\left(1+\lambda_{\rho-}\right)^{2}}-\frac{2 \widetilde{V}_{G}\left(\widetilde{V}_{G}+\lambda_{G}\right)}{1+\lambda_{\rho-}}+\frac{g^{2} \mu_{\sigma-}^{2}}{\left(g^{2}+\lambda_{\sigma^{-}}\right)^{2}}\right] \\
& +\frac{1}{2 \epsilon_{L} \beta} \sum_{\omega_{n}, a} \ln \left[\frac{\epsilon_{L} \beta}{\pi}\left[\mathcal{K}_{a}\left(\omega_{n}\right)+\lambda_{a}\right]\right] \\
& -\frac{1}{2 \epsilon_{L} \beta} \sum_{\omega_{n}, a} \frac{\lambda_{a}}{\lambda_{a}+\mathcal{K}_{a}\left(\omega_{n}\right)} \\
& -4 \tilde{u}\left\{\cos \left[\frac{\pi\left(\widetilde{V}_{G}+\lambda_{G}\right)}{1+\lambda_{\rho-}}\right] \cos \left(\frac{\pi \mu_{\rho+}}{\lambda_{\rho+}}\right) \cos \left(\frac{\pi \mu_{\sigma-}}{g^{2}+\lambda_{\sigma-}}\right)\right. \\
& \times \cos \left(\frac{\pi \mu_{\sigma+}}{\lambda_{\sigma+}}\right)+\sin \left[\frac{\pi\left(\widetilde{V}_{G}+\lambda_{G}\right)}{1+\lambda_{\rho-}}\right] \sin \left(\frac{\pi \mu_{\rho+}}{\lambda_{\rho+}}\right) \\
& \left.\times \sin \left(\frac{\pi \mu_{\sigma-}}{g^{2}+\lambda_{\sigma-}}\right) \sin \left(\frac{\pi \mu_{\sigma+}}{\lambda_{\sigma+}}\right)\right\} \\
& \times \exp \left[-\frac{1}{4 \epsilon_{L} \beta} \sum_{\omega_{n}, a} \frac{1}{\mathcal{K}_{a}\left(\omega_{n}\right)+\lambda_{a}}\right],
\end{aligned}
$$

with $a=\rho \pm, \sigma \pm$. Equation (A2) is minimized when $\lambda_{\rho / \sigma \pm}$ $\equiv \lambda$ and either

$$
\sin \left(\frac{\pi \mu_{\rho+}}{\lambda}\right)=\sin \left(\frac{\pi \mu_{\sigma+}}{\lambda}\right)=0,
$$

or

$$
\cos \left(\frac{\pi \mu_{\sigma+}}{\lambda}\right)=\cos \left(\frac{\pi \mu_{\rho+}}{\lambda}\right)=0 .
$$

Equation (A3) leads to the following coupled equations for $\lambda, \mu_{\sigma^{-}}$, and the charge $Q$ :

$$
\frac{\pi \mu_{\sigma-}}{g^{2}+\lambda}=\frac{1}{g^{2}} \sqrt{\gamma^{\prime 2} \lambda^{\Delta+1} e^{-2 I^{\prime}(\lambda)} \cos ^{2}\left(\frac{\pi Q}{2}\right)-\lambda^{2}},
$$




$$
\begin{gathered}
\lambda=\zeta \gamma^{\prime} \lambda^{(\Delta+1) / 2} e^{-I^{\prime}(\lambda)} \cos \left(\frac{\pi Q}{2}\right) \cos \left(\frac{\pi \mu_{\sigma-}}{g^{2}+\lambda}\right), \\
\cos ^{2}\left(\frac{\pi Q}{2}\right)=\left[1+\pi^{2}\left(\frac{Q / 2-\widetilde{V}_{G}}{\lambda}\right)^{2}\right]^{-1}
\end{gathered}
$$

By inspection and confirmed by numerical analysis, these equations are satisfied when $\mu_{\sigma-}=0$ and $\lambda$ is a solution to

$$
\begin{aligned}
\lambda= & \zeta \gamma^{\prime} \lambda^{(\Delta+1) / 2} e^{-I^{\prime}(\lambda)} \\
& \times \cos \left[\pi \widetilde{V}_{G}+\eta \sqrt{\gamma^{\prime 2} \lambda^{\Delta+1} e^{-2 I^{\prime}(\lambda)}-\lambda^{2}}\right] .
\end{aligned}
$$

This decoupled equation for $\lambda$ can be used in conjunction with Eq. (A5) to obtain Eq. (18) for $Q$ in terms of $\lambda$.

Equation (A4) yields a set of coupled equations identical to Eqs. (A5)-(A7) except that the cosines are replaced by sines. In this case, one can not obtain a decoupled equation for $\lambda$. Instead, we first use the analog of Eq. (A7) to write $\lambda$ as a function of the charge. We then use the remaining two equations to obtain a decoupled equation for the charge, Eq. (21).

\section{APPENDIX B: VARIATIONAL RESULTS IN THE LOW BACKSCATTERING LIMIT}

For completeness, in this appendix we review the variational solution in the limit of near-perfect contacts where the technique yields unphysical results. In this limit the failure of the technique is due to the method neglecting analytic terms in the free energy, which in this case provide non-negligible contributions. In the spinless case, the variational results are unphysical when $g_{L} \geqslant 1 / 2$ and $\gamma<\gamma_{c}$. As Fig. 5 illustrates, in this regime $\sigma$ exhibits two peaks per period. When $g_{L} \geqslant 1$ this structure arises because the free energy is minimized when $\lambda=0$ in the region of $\widetilde{V}_{G}$ between those peaks. As $\gamma$ decreases, the width of this region increases and the peaks in $\sigma$ spread farther apart until $\lambda=0$ everywhere and the peaks disappear entirely. This can be understood by noting that in the limit $\gamma \ll 1$ the system essentially reduces to the singleimpurity problem discussed in Sec. III for which $\lambda=0$ is the only solution when $g_{L} \geqslant 1$. In this limit the variational method thus predicts that $Q=\widetilde{V}_{G}$. While this is consistent with renormalization group arguments when $g_{L}>1$, in the case of Fermi liquid leads $\left(g_{L}=1\right)$ one would expect to see small Fabry-Perot oscillations in the charge as the gate voltage varies. These Fabry-Perot oscillations have been observed in the conductance of single-walled nanotubes with near-perfect Ohmic contacts, ${ }^{3}$ and were also captured by a perturbative calculation of the charge in Sec. IV A.
For $g_{L}<1$, the free energy is minimized by a nontrivial $\lambda$ for the entire range of gate voltages. This solution can be obtained analytically by first noting that $\lambda \ll 1$ when $\gamma \ll 1$ so that the dominant contribution to the integral in $I(\lambda)$ comes from the region of small $x$ where $x \lesssim g$. To approximate $I(\lambda)$, we therefore cut off the integral at $x=g$ and expand $\mathcal{K}_{ \pm}\left(2 \pi \epsilon_{L} x\right)$ to first order in $x$. For $\widetilde{V}_{G}$ away from a halfinteger, the square-root term in Eq. (10) can be dropped, allowing determination of $\lambda$. The resulting charge when $\widetilde{V}_{G}$ is away from a half-integer is

$$
Q-\widetilde{V}_{G} \propto \eta \gamma^{1 /\left(1-g_{L}\right)}\left|\sin \left(\pi \widetilde{V}_{G}\right)\right|\left|\cos \left(\pi \widetilde{V}_{G}\right)\right|^{g_{L} /\left(1-g_{L}\right)} .
$$

When $1 / 2<g_{L}<1$, Eq. (B1) predicts an unphysical doublepeak structure in $\sigma$ similar to that shown in Fig. 5(b), while for $g_{L} \leqslant 1 / 2$, Eq. (B1) predicts oscillations in $\sigma$ with a single peak per period. An analysis of the charge at half-integer values of $\widetilde{V}_{G}$, however, reveals that the charge is discontinuous at these values when $g_{L}<1 / 2$. If we correct for this artifact, then the variational technique reproduces the expected Fabry-Perot oscillations in $\sigma$ when $g_{L} \leqslant 1 / 2$.

In the spinful case, the variational technique yields unphysical results for the charge when $\gamma^{\prime} \lesssim \gamma_{c}^{\prime}$. Here, $\gamma_{c}^{\prime}$ corresponds to the value of $\gamma^{\prime}$ below which the charge is given for all $\widetilde{V}_{G}$ by Eq. (18), which has a nearly identical structure to Eq. (12) governing the charge in the spinless case. The behavior of $\sigma$ in this regime is therefore very similar to that in the spinless case, and the method once again predicts an unphysical double-peak structure in $\sigma$ as depicted in Fig. 9. The main difference here is that this failure occurs for all $g_{L}$ since the effective interaction parameter in the leads is $\left(g_{L}\right.$ $+1) / 2>1 / 2$. For $g_{L} \geqslant 1$, the region between the peaks in $\sigma$ where the free energy is minimized by $\lambda=0$ increases as $\gamma^{\prime}$ is lowered. Eventually, $\lambda=0$ everywhere and the peaks disappear altogether.

In the limit $\gamma^{\prime} \ll 1$, the charge can be obtained analytically by invoking similar approximations that were made in the spinless case. We again find only trivial solutions for $\lambda$ when $g_{L} \geqslant 1$ so that $Q=2 \widetilde{V}_{G}$. For $g_{L}<1$, however, the free energy is minimized by a nontrivial value of $\lambda$, and the charge away from half-integer values of $\widetilde{V}_{G}$ is

$Q-2 \widetilde{V}_{G} \propto 2 \eta \gamma^{\prime 2 /\left(1-g_{L}\right)}\left|\sin \left(\pi \widetilde{V}_{G}\right)\right|\left|\cos \left(\pi \widetilde{V}_{G}\right)\right|^{\left(1+g_{L} /\left(1-g_{L}\right)\right)}$.

It follows from Eq. (B2) that $\sigma$ exhibits an unphysical double-peak structure for all $g_{L}<1$. When $\widetilde{V}_{G}$ equals a half integer we find that $\lambda=0$ is the only solution for any $g_{L}$ $<1$ so that the charge is a continuous function of $\widetilde{V}_{G}$.

\footnotetext{
*Electronic address: aliceaj@physics.ucsb.edu

†Electronic address: cristina@physics.ucsb.edu

†Electronic address: balents@ physics.ucsb.edu

${ }^{\S}$ Electronic address: mpaf@kitp.ucsb.edu

${ }^{1}$ W. Liang, M. Bockrath and H. Park, Phys. Rev. Lett. 88, 126801 (2002)

${ }^{2}$ S.J. Tans, M.H. Devoret, H. Dai, A. Thess, R.E. Smalley, L.J.
}

Geerligs, and C. Dekker, Nature (London) 386, 474 (1997).

${ }^{3}$ W. Liang, M. Bockrath, D. Bozovic, J.H. Hafner, M. Tinkham, and H. Park, Nature (London) 411, 665 (2001); J. Nygard and D.H. Cobden, Electronic Correlations: From Meso- to Nano-Physics, edited by T. Martin, G. Montambaux and J. Tran Thanh Van (EDP Sciences, Les Ulis, France 2001) [cond-mat/0105289]. 
${ }^{4}$ A. Rubio, S.P. Apell, L.C. Venema, and C. Dekker, Eur. Phys. J. B 17, 301 (2000); Z. Yao, C.L. Kane, and C. Dekker, Phys. Rev. Lett. 84, 2941 (2000); C. Dekker, Phys. Today 52 (5), 22 (1999); M. Bockrath, D.H. Cobden, J. Lu, A.G. Rinzler, R.E. Smalley, L. Balents, and P.L. McEuen, Nature (London) 397, 598 (1999); Z. Yao, H. Postma, L. Balents, and C. Dekker, ibid. 402, 273 (1999); H. Postma, M. de Jonge, Z. Yao, and C. Dekker, Phys. Rev. B 62, R10 653 (2000).

${ }^{5}$ M. Bockrath, D.H. Cobden, P.L. McEuen, N.G. Chopra, A. Zettl, A. Thess, and R.E. Smalley, Science 275, 1922 (1997).

${ }^{6}$ J. Nygard, D.H. Cobden, M. Bockrath, P.L. McEuen, and P.E. Lindelof, Appl. Phys. A: Mater. Sci. Process. 69, 297 (1999).

${ }^{7}$ C.S. Peca, L. Balents, and K.J. Wiese, Phys. Rev. B 68, 205423 (2003).

${ }^{8}$ R.P. Feynman, Phys. Rev. 97, 660 (1955).

${ }^{9}$ R.P. Feynman and A.R. Hibbs, Quantum Mechanics and Path Integrals (McGraw-Hill, New York, 1965).

${ }^{10}$ C. Kane, L. Balents, and M.P.A. Fisher, Phys. Rev. Lett. 79, 5086 (1997).

${ }^{11}$ R. Egger and A.O. Gogolin, Phys. Rev. Lett. 79, 5082 (1997).

${ }^{12}$ S. Tomonaga, Prog. Theor. Phys. 5, 544 (1950); J.M. Luttinger, J. Math. Phys. 4, 1154 (1963); F.D.M. Haldane, J. Phys. C 14,
2585 (1981); Phys. Rev. Lett. 47, 1840 (1981).

${ }^{13}$ K.A. Matveev, Phys. Rev. B 51, 1743 (1995); A. Furusaki and K.A. Matveev, Phys. Rev. Lett. 75, 709 (1995); I.L. Aleiner, P.W. Brouwer, and L.I. Glazman, Phys. Rep. 358, 309 (2002); Thomas Gramespacher and K.A. Matveev, Phys. Rev. Lett. 85, 4582 (2000).

${ }^{14}$ W. Iwo Babiaczyk and Bogdan R. Bulka, Acta Phys. Pol. A 102, 597 (2002); Calin Buia, Alper Buldum, and Jian Ping Lu, Phys. Rev. B 67, 113409 (2003).

${ }^{15}$ J.-S. Caux, H. Saleur, and F. Siano, Nucl. Phys. B 672, 411 (2003); A. Koutouza, F. Siano, and H. Saleur, J. Phys. A 34, 5497 (2001); A. LeClair and A.W.W. Ludwig, Nucl. Phys. B 549, 546 (1999); P. Fendley, A.W.W. Ludwig, and H. Saleur, Phys. Rev. B 52, 8934 (1995).

${ }^{16}$ C.L. Kane and M.P.A. Fisher, Phys. Rev. Lett. 68, 1220 (1992); Phys. Rev. B 46, 15233 (1992).

${ }^{17}$ M.P.A. Fisher and L.I. Glazman, in Mesoscopic Electron Transport of NATO, Advanced Studies Institute, Series E: Applied Sciences Vol. 345 edited by L.L. Sohn, L.P. Kouwenhoven, and G. Schon (Kluwer Academic Publishing, Dordrecht, 1997), p. 331. 